\title{
Rheology of Styrene-Butadiene Diblock Copolymers Dissolved in Selective Solvent: Dependence on Molecular Dimension
}

\author{
Hiroshi WATANABE and Tadao KoTAKA \\ Department of Macromolecular Science, Faculty of Science, \\ Osaka University, Toyonaka, Osaka 560, Japan
}

(Received June 3, 1982)

\begin{abstract}
The rheology of solutions of a series of styrene-butadiene diblock copolymers dissolved in a highly selective solvent, $n$-tetradecane, was examined in relation to the molecular dimension of the block copolymers. Micelles with precipitated S cores and dissolved $\mathbf{B}$ cilia are formed in the solution. Above a certain concentration and below a certain temperature, micelles in the solution are spontaneously arranged to form a regular three-dimensional array referred to as a macrolattice. Solutions having macrolattice structures exhibit nonlinear viscoelastoplastic flow, and those having no macrolattices exhibit linear viscoelastic flow. The critical concentration, at which transitions in rheology and morphology occur, may be predicted from the structural characteristics of the micelles, i.e., their size and number of molecules making up a micelle. The relationship of dynamic moduli and the molecular dimensions was examined in detail. The shorter the length of dissolved B blocks, the larger the storage modulus and the smaller the loss modulus. Two mechanisms, being the delayed relaxational motions of the dissolved $\mathbf{B}$ blocks and deformation of the macrolattice itself, seem responsible for the stress in the solution. The mechanism of the lattice disordering caused by heat is also discussed. A diffuse interphase between the S core and B cilia seems to play a key role in the lattice disordering.
\end{abstract}

KEY WORDS Styrene-Butadiene Diblock Copolymer / Block Copolymer Solution / Solution Rheology / Microphase Separation / Superstructure / Liquid Crystalline System / Nonlinear Viscoelastoplasticity / Plastic Flow /

Solutions of styrene-butadiene (SB) and other diblock copolymers dissolved in selective solvents, in which only one of the blocks is soluble and the other insoluble, show peculiar rheology. Such solutions often show thixotropic stress decay under steady shear and possess plasticity. ${ }^{1-3}$ In such diblock copolymer solutions, the insoluble blocks precipitate and microphase separation occurs even at an infinitely high dilution to form micelles. ${ }^{4}$ One might imagine that these micelles themselves govern the peculiar rheology. However, such solutions exhibit neither thixotropy nor plasticity when the solutions are relatively dilute. ${ }^{1,5}$

Recently, we examined the plasticity of solutions of a commercially available SB diblock copolymer dissolved in selective solvents such as $n$-tetradecane and dibutyl-phthalate in greater detail. ${ }^{5-7}$ When the copolymer (coded as cSB, Phillips Petroleum
Solprene 1205) of an average molecular weight $M_{w}$ of $52 \times 10^{3}$ and $\mathrm{S}$ content of $29.5 \mathrm{wt} \%$ was dissolved in $n$-tetradecane that dissolves only B-blocks, micelles with $\mathrm{S}$ cores and $\mathrm{B}$ cilia were formed in the system. Above a certain concentration and below a certain temperature, these micelles became arranged on a simple cubic type regular three-dimensional array. This kind of solution is referred to as a micelle system and the regular structure, a macrolattice. When the system was moderately heated or diluted, the macrolattice lost its long-range order and the micelles became randomly dispersed. A detailed small-angle X-ray scattering study showed that this structural transition corresponded to the transition found in the rheological properties. ${ }^{6-8}$ The system having a macrolattice exhibited nonlinear viscoelastoplasticity, while the system showing linear viscoelasticity as the macrolattice was 
lost. That is, the peculiar rheology of these micelle systems is a result of their liquid crystalline nature.

In the previous paper, ${ }^{6}$ we proposed an idea for the force leading to the formation of a macrolattice in solution. The equilibrium domain morphology in a bulk block copolymer is determined by a thermodynamic force. ${ }^{9,10}$ This force leads the system to an optimum state where the density of the block segments is automatically adjusted as uniformly as possible, and at the same time, the conformational entropy of the blocks becomes as large as possible. A theory of bulk block copolymers incorporating this thermodynamic force was first developed by Meier ${ }^{9}$ and further elaborated by Helfand and Wasserman. ${ }^{10}$ They treated the incompressible limit where no density fluctuation is allowed and successfully predicted the domain size and shape as a function of molecular weight and composition of the block copolymer molecules. In an SB micellar solution, the driving force of the macrolattice formation is similar to this thermodynamic force acting in bulk block copolymers. An essential difference between the solution and the bulk is that in the micellar solution, solvent molecules automatically compensate for density fluctuation. However, there remains the spatial fluctuation of the concentration of the dissolved blocks. The thermodynamic force generated by the local concentration gradient seems sufficient to drive the micelles to form the macrolattice. This idea is supported by an experiment ${ }^{11,12}$ in which the micelle system loses its plasticity when low molecular weight homopolybutadiene $(\mathrm{hB})$ is added. The $\mathrm{hB}$ molecules act as a buffer to suppress the concentration fluctuation and destroy the regular macrolattice of the micelles.

This scheme may be further tested by examining the relation of the critical concentration and temperature to the molecular dimension of the block copolymer molecules. For the thermodynamic force to become active, the ciliary B chains of neighboring micelles must overlap to some extent so that the concentration gradient is generated on displacing micelles from their equilibrium position in the lattice. Knowing the molecular dimension of the block copolymer molecules and the size of the micelles involved, it is possible to predict the critical concentration at which the macrolattice formation and hence the transition of the rheological behavior take place. We prepared two series of five SB diblock samples in which each series had the same S-block but different B-blocks, and examined their rheology in $n$-tetradecane. The effects of the block lengths on the critical concentration and temperature at which structural and rheological transitions take place were carefully examined.

\section{EXPERIMENTAL}

\section{Materials}

Five SB diblock copolymer samples were prepared by an anionic living polymerization method. First, a styrene monomer was polymerized in benzene with $s$-butyllithium at room temperature. After $48 \mathrm{~h}$ polymerization, the whole batch of living polystyrene was divided up and placed in two or three reaction vessels. A prescribed amount of butadiene monomer was distilled into each vessel and allowed to polymerize with the living polystyrene at room temperature. After 5 days, the reaction was terminated with methanol. An antioxidant, butylhydroxytoluene (BHT), of about $0.1 \mathrm{wt} \%$ of the product SB was added to the benzene solution. The whole solution was freeze dried to obtain the SB block copolymer.

The molecular characteristics of the samples were determined by a gel permeation chromatograph (GPC: Toyo Soda Ltd., Model HLC-801 A) provided with a triple detector system consisting of a built-in refractometer, a UV-absorption detector (Toyo Soda Ltd., Model UV-8) and a low-angle laser light scattering (LALLS) photometer (Toyo Soda Ltd., Model LS-8) connected in series. A chloroform carrier and commercially available PS elution standards (Toyo Soda Ltd., TSK PS's) were used to determine $\mathrm{Mn}$ and the molecular weight distribution. Table I shows the sample codes and the characteristics of these copolymers. The SB1 and SB2 were prepared from one batch of living PS,

Table I. Characteristics of SB diblock copolymer samples

\begin{tabular}{crrcc}
\hline Code & $10^{-4} M_{n}$ & $M_{w} / M_{n}$ & $\begin{array}{c}\text { PS cont. } \\
10^{-4} M_{n} \text { of } \\
\text { S block }\end{array}$ \\
& & & wt $\%$ & \\
\hline SB1 & 6.6 & 1.06 & 30 & 2.0 \\
SB2 & 12.0 & 1.07 & 16 & 2.0 \\
SB3 & 13.4 & 1.07 & 24 & 3.2 \\
SB4 & 19.2 & 1.08 & 17 & 3.2 \\
SB5 & 29.4 & 1.10 & 11 & 3.2 \\
\hline
\end{tabular}


and the SB3, SB4, and SB5 from another batch. Therefore, the S-blocks of SB1 and SB2 had the same characteristics as well as those of SB3, SB4, and SB5, as shown in Table I.

The systems examined were solutions of these copolymers dissolved in $n$-tetradecane (coded as C14, Nakarai Chemicals Co., guaranteed grade). A typical solution was prepared by mixing a prescribed amount of the SB copolymer and solvent with an excess of methylene chloride, which was subsequently evaporated to obtain a homogeneous but opaque solution of the desired concentration. ${ }^{2}$ The volume fraction of the $(\mathrm{B}+\mathrm{C} 14)$ phase (the matrix phase) was much larger than that of the $S$ phase and thus the micelles with spherical $S$ cores and $\mathrm{B}$ cilia are formed in the $\mathrm{C} 14$ solution. ${ }^{13}$ All the systems examined were coded as solute code/solvent code ( $w \mathrm{t} \%$ of the solute). For example, SB1/C14(6) represents a $6 \mathrm{wt} \%$ solution of the SB1 copolymer dissolved in $n$-tetradecane.

\section{Methods}

Rheological Measurement. Steady flow and dynamic measurements were carried out with a laboratory rheometer mounted with a coaxial cylinder (Couette) assembly. ${ }^{6}$ The radii $R_{1}$ and $R_{2}$ of the bob and cup were 12.5 and $15.0 \mathrm{~mm}$, respectively, and the height $h$ of the bob was $70 \mathrm{~mm}$.

When the concentration of SB exceeded a certain critical value, the solution showed nonlinear viscoelastoplasticity. ${ }^{5-7}$ The dynamic stress profile against a sinusoidal strain input was not sinusoidal and contained higher-order odd harmonics. To analyze this behavior, we assumed a constitutive equation of Fourier series type defined by eq 1, and the nonlinear dynamic moduli $G_{j}{ }^{\prime}$ and $G_{j}{ }^{\prime \prime}$ were determined numerically.

$$
\begin{aligned}
& \gamma=\gamma_{0} \sin \omega t, \\
& \sigma=\gamma_{0} \sum_{j}\left[G_{j}^{\prime} \sin j \omega t+G_{j}^{\prime \prime} \cos j \omega t\right]
\end{aligned}
$$

The details are described elsewhere ${ }^{5,6}$ and thus need not appear here. Steady flow data are summarized as the apparent shear rate $\dot{\gamma}_{\text {app }}=2 R_{2}^{2} \dot{\Omega} /\left(R_{2}{ }^{2}-R_{1}{ }^{2}\right)$ versus steady shear stress at the wall of the bob $R_{1}$, where $\dot{\Omega}$ denotes the angular velocity of the cup rotation.

Small Angle X-Ray Scattering Measurement. Small angle X-ray scattering (SAXS) studies were carried out by Mr. Shibayama and Dr. Hashimoto at Prof. Kawai's Laboratory, Department of Polymer Chemistry, Kyoto University, to determine the structural characteristics of the micelle systems. The SAXS apparatus made possible high speed measurements of SAXS profiles with a linear position sensitive detector (PSD) and a $12 \mathrm{~kW}$ rotating anode X-ray generator (Rigaku Denki, RU-a). ${ }^{14}$ $\mathrm{Cu}-K \alpha$ radiation $(\lambda=1.54 \AA)$ was used as the incident X-ray beam which was monochromatized by a graphite monochrometer and analyzed by a pulse height analyzer. The camera distance from the sample specimen to the PSD was set at $1154 \mathrm{~mm}$. The effective length of the PSD (about $50 \mathrm{~mm}$ ) was resolved into 256 channels with one channel corresponding to a scattering angle of $0.7 \mathrm{~min}$ of an arc. The raw data obtained was corrected for absorption, parastic scattering, scattering from the cell enclosing the solution, background scattering arising from the thermal diffuse scattering, collimation errors both slit-width and slit-length directions, and nonuniformity of the sensitivity of the PSD to give desmeared SAXS profiles. The details are described elsewhere. ${ }^{8,14}$

\section{RESULTS AND DISCUSSION}

\section{Critical Concentration versus Molecular Dimension}

Figure 1 shows the steady flow behavior of SB1/C14 micelle systems at various concentrations at $25^{\circ} \mathrm{C}$. The transition from plastic to viscous (or viscoelastic) flow takes place between 7 and $6 \mathrm{wt} \%$ for this system. This transition also appears in the dynamic behavior. Figure 2 shows the frequency dependence of the nonlinear moduli $G_{j}{ }^{\prime}$ and $G_{j}{ }^{\prime \prime}$ defined by eq 1 of the SB1/C14(6) and SB1/C14(7) systems at $25^{\circ} \mathrm{C}$. The $\mathrm{SB} 1 / \mathrm{C} 14(7)$ system shows typical nonlinear viscoelastoplasticity: ${ }^{5-7}$ (i) The higher odd harmonics $\left(G_{3}{ }^{\prime}\right.$ and $\left.G_{3}{ }^{\prime \prime}\right)$ contribute considerably to the overall stress. (ii) The fundamental harmonics $\left(G_{1}{ }^{\prime}\right.$ and $\left.G_{1}{ }^{\prime \prime}\right)$ show plateaus against frequency. (iii) The ratio of the amplitudes $G_{j} / G_{1}$ of the $j$-th to the fundamental harmonics defined as

$$
G_{j} / G_{1}=\left[\left(G_{j}{ }^{\prime 2}+G_{j}{ }^{\prime \prime 2}\right) /\left(G_{1}{ }^{\prime 2}+G_{1}{ }^{\prime \prime 2}\right)\right]^{1 / 2}
$$

$(j=3,5)$ is nearly $10 \%$ for $j=3$ and decreases with increasing frequency. The decrease in $G_{j} / G_{1}$ is also typical of a micellar system having a macrolattice structure. On the other hand, the SB1/C14(6) system shows linear and Newtonian behavior, i.e., the 
ratio $G_{j} / G_{1}$ is zero for all frequencies.

Steady flow and dynamic measurements were carried out also for other block copolymer systems, and the critical concentration $c_{\mathrm{R}}{ }^{*}$ above which the

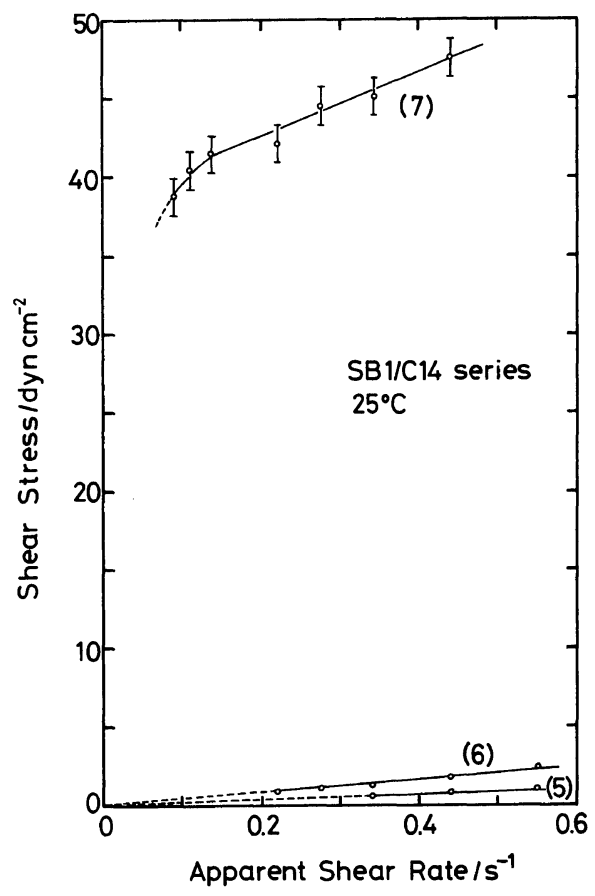

Figure 1. Steady flow behavior of the SB1/C14 solutions at concentrations of 5,6 , and $7 \mathrm{wt} \%$ obtained at $25^{\circ} \mathrm{C}$. system exhibited nonlinear viscoelastoplasticity was determined. Table II shows the values of $c_{\mathrm{R}}{ }^{*}$.

The transition shown in Figures 1 and 2 corresponds to the onset of the macrolattice formation, ${ }^{6}$ and $c_{\mathrm{R}}{ }^{*}$ should be estimated from the structural and morphological characteristics of the micelles. Figure 3 shows our underlying principle for this estimation. The driving force of the macrolattice formation seems to become effective when the micelles are arranged on a simple cubic macrolattice and their ciliary $B$ chains begin to contact and overlap to some extent. We do not know what degree of overlapping is necessary to sustain the macrolattice and generate the yield stress detected by our rheological measurements. We estimated two concentrations $c_{1}{ }^{*}$ and $c_{2}{ }^{*}$ as illustrated in

Table II. Comparison of the critical concentrations $c_{\mathrm{R}}{ }^{*}$ determined from rheological transition with $c^{*}$ estimated from morphological behavior

\begin{tabular}{lllll}
\hline & & \multicolumn{2}{c}{$c^{*}$ Calculated } \\
\cline { 3 - 4 } \cline { 4 - 5 } System & $c_{\mathrm{R}}{ }^{*}$ & & $c_{1}{ }^{*}$ & $c_{2}{ }^{*}$ \\
\cline { 3 - 5 } & $\mathrm{wt} \%$ & & $\mathrm{wt} \%$ & $\mathrm{wt} \%$ \\
\hline $\mathrm{SB} 1 / \mathrm{C} 14$ & $6-7$ & & 1.5 & 3.8 \\
$\mathrm{SB} 2 / \mathrm{C} 14$ & $2-3$ & & 1.0 & 2.5 \\
$\mathrm{SB} 3 / \mathrm{C} 14$ & $3-4$ & & 0.9 & 2.3 \\
$\mathrm{SB} 4 / \mathrm{C} 14$ & $1-2$ & & 0.8 & 2.2 \\
$\mathrm{SB} 5 / \mathrm{C} 14$ & $1.2-1.5$ & 0.6 & 1.6 \\
\hline
\end{tabular}

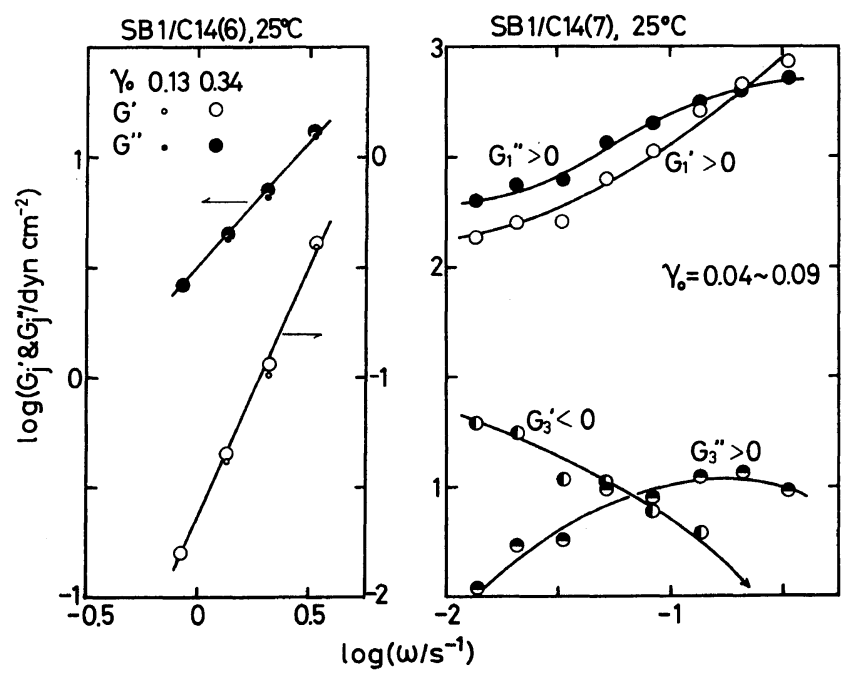

Figure 2. Dynamic behavior of the SB1/C14(6) and SB1/C14(7) systems at $25^{\circ} \mathrm{C}$. 


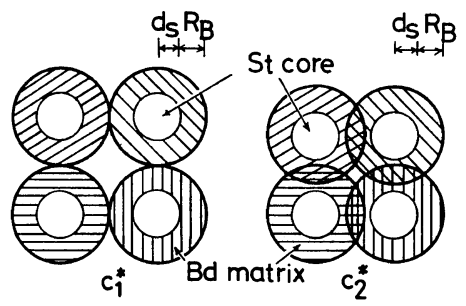

Figure 3. A schematic representation of the two critical concentrations $c_{1}{ }^{*}$ and $c_{2}{ }^{*}$ : The $c_{1}{ }^{*}$ is the concentration at which the micelles arranged on a simple cubic lattice just begin to contact. The $c_{2}{ }^{*}$ is the concentration at which the volume of the solution equally divided among the micelles equals the volume of the micelle itself, i.e., the critical threshold. The parameters $d_{\mathrm{S}}$ and $R_{\mathrm{B}}$ denote the radius of the $\mathrm{S}$ core and thickness of the $\mathrm{B}$ block layer, respectively.

Figure 3. $c_{1}{ }^{*}$ is the concentration at which two neighboring micelles just begin to contact with each other. On the other hand, $c_{2}{ }^{*}$ is the concentration at which the volume of the solution is divided equally, each part being equal to the volume of one micelle. $c_{2}{ }^{*}$ corresponds to the critical threshold as defined by de Gennes ${ }^{15}$ from dilute to semidilute regime of the micelle system. In other words, the space between the cores (which are on the lattice points) just begins to be filled uniformly with ciliary $B$ chains. Both concentrations, $c_{1}{ }^{*}$ and $c_{2}{ }^{*}$, were evaluated from the structural characteristics of a micelle as

$$
c_{i}^{*}=\frac{100 q_{i}}{\rho} \frac{N_{m} M_{\mathrm{SB}}}{\left(R_{\mathrm{B}}+d_{\mathrm{S}}\right)^{3} N_{\mathrm{A}}} \quad(\mathrm{wt} \%), \quad i=1,2
$$

where $N_{\mathrm{A}}$ is the Avogadro number, $\rho$ the density of the system, $M_{\mathrm{SB}}$ the molecular weight of the SB sample examined, $R_{\mathrm{B}}$ the thickness of the B block layer, $d_{\mathrm{S}}$ the radius of the $\mathrm{S}$ core, and $N_{m}$ the number of the SB molecules belonging to one micelle. The numerical parameters $q_{i}(i=1,2)$ are given as

$$
q_{1}=\frac{1}{8}, \quad q_{2}=\frac{3}{4 \pi}
$$

The parameters $d_{\mathrm{S}}$ and $N_{m}$ could be estimated from the SAXS data. Figure 4 shows the desmeared SAXS profiles of $10 \mathrm{wt} \% \mathrm{C} 14$ solutions of the SB samples obtained at $25^{\circ} \mathrm{C}$. There appear scattering maxima attributed to interparticle interference (indicated by arrows in Figure 4) as well as a scattering maximum $\tilde{\theta}$ from an isolated domain (marked by a diamond-shaped arrow). The Bragg spacing $D$ of the macrolattice was estimated from the position of



Figure 4. The desmeared SAXS profiles of the $10 \mathrm{wt} \%$ C14 solutions of the SB1 to SB5 block copolymers obtained at $25^{\circ} \mathrm{C}$.

the $n$-th order interparticle interference scattering peak $\theta_{n}$ as

$$
D=\frac{\lambda}{2 \sin \left(\theta_{n} / n\right)}
$$

where $\lambda$ denotes the wavelength of the incident $\mathrm{X}$ ray beam. The Bragg spacing $D$ is so large for the $\mathrm{SB} 2$ to $\mathrm{SB} / \mathrm{C} 14(10)$ systems that the scattering peaks shift to the small angle region and the second order peak is not well resolved. In this regard, the first order peak of the SB5/C14(10) system, which has the largest $D$, appears at an angle too small to allow detection. From the value of $D$, the number of SB molecules per micelle $N_{m}$ was estimated for the $10 \mathrm{wt} \%$ solution as

$$
N_{m}=0.1 \rho D^{3} N_{\mathrm{A}} / M_{\mathrm{SB}}
$$

On the other hand, the radius $d_{\mathrm{S}}$ of the $\mathrm{S}$ core was estimated from the position $\tilde{\theta}$ of the scattering peak attributed to an isolated domain as ${ }^{8}$ 
Table III. Structural characteristics of $10 \mathrm{wt} \%$ micelle systems

\begin{tabular}{|c|c|c|c|c|c|c|}
\hline \multirow[t]{2}{*}{ System } & \multicolumn{2}{|c|}{$\frac{2 \theta_{n}}{\min }$} & \multirow{2}{*}{$\frac{D}{\bar{\AA}}$} & \multirow[t]{2}{*}{$N_{m}$} & \multirow{2}{*}{$\frac{2 \tilde{\theta}}{\min }$} & \multirow{2}{*}{$\frac{d_{\mathrm{S}}}{\AA}$} \\
\hline & 1st & $3 \mathrm{rd}$ & & & & \\
\hline $\mathrm{SB} 1 / \mathrm{C} 14$ & 9.48 & 18.16 & 505 & 92 & 47.38 & 103 \\
\hline $\mathrm{SB} 2 / \mathrm{C} 14$ & 8.26 & 15.08 & 608 & 90 & 46.67 & 104 \\
\hline SB3/C14 & 7.90 & 14.50 & 632 & 88 & 38.77 & 125 \\
\hline SB4/C14 & 6.82 & 12.34 & 743 & 100 & 37.33 & 130 \\
\hline $\mathrm{SB} 5 / \mathrm{C} 14$ & - & 10.77 & 851 & 98 & 35.90 & 135 \\
\hline
\end{tabular}

$$
d_{\mathrm{S}}=5.765 \lambda /(4 \pi \sin \tilde{\theta})
$$

Table III summarizes the SAXS data indicating the values of $\theta_{n}, D, N_{m}, \tilde{\theta}$ and $d_{\mathrm{S}}$ for the $10 \mathrm{wt} \% \mathrm{SB} / \mathrm{C} 14$ solutions. The values of $N_{m}$ estimated from the positions of the first and third scattering peaks slightly differed with each other. Because the experimental error became larger as the peak shifted to small angle, the value of $N_{m}$ estimated from the position of the first peak was adopted in Table III. It should be noted that the radius $d_{\mathrm{S}}$ is proportional to the square of $M_{\mathrm{S}}$, as pointed out by Meier ${ }^{9}$ and Helfand..$^{10}$ In SB diblock copolymer solutions, as with other diblock copolymers ${ }^{4}$ dissolved in a selective solvent, the structural characteristics of the micelle depend little on the concentration of the SB. ${ }^{8}$ The values of $d_{\mathrm{S}}$ and $N_{m}$ for the $10 \mathrm{wt} \%$ solution (which is higher than the $c_{\mathrm{R}}{ }^{*}$ ) may be used for estimating $c_{1}{ }^{*}$ and $c_{2}{ }^{*}$.

The estimation of the thickness of the $\mathrm{B}$ layer $R_{\mathrm{B}}$ is most difficult. The $R_{\mathrm{B}}$ can be approximated as the root mean square end-to-end distance of the $B$ block. The unperturbed root mean square end-toend distance $\left\langle r^{2}\right\rangle$ is given as ${ }^{16}$

$$
\left\langle r^{2}\right\rangle_{0}^{1 / 2}=b n_{\mathrm{B}}^{0.5}
$$

where $n_{\mathrm{B}}$ and $b$ denote the degree of polymerization and the monomer size (Kuhn length) of the B block. On the other hand, when the B chain is isolated and strongly expanded by intramolecular excluded volume interactions, the root mean square end-to-end distance $\left\langle r^{2}\right\rangle^{1 / 2}$ is given as ${ }^{17}$

$$
\left\langle r^{2}\right\rangle^{1 / 2}=b n_{\mathrm{B}}{ }^{0.6}
$$

In our moderately concentrated system, the $B$ blocks overlap with each other and the excluded volume effect is modestly screened. The conformational state of such B blocks may be described as an intermediate between eq 8 and 9, i.e., by the blob model proposed by de Gennes. ${ }^{15} \mathrm{~A}$ blob consists of $g$ monomers, and the size of the blob is $\xi$. The scaling forms of $g$ and $\xi$ are given as ${ }^{15}$

$$
g \cong \phi^{-5 / 4}, \quad \xi \cong b \phi^{-3 / 4}
$$

where $\phi$ denote the volume fraction of the B segments. The whole B block in the semidilute state is regarded as an ideal chain consisting of $n_{\mathrm{B}} / g$ blobs with the size $\xi$. The $R_{\mathrm{B}}$ is now given as

$$
R_{\mathrm{B}}=\xi\left(n_{\mathrm{B}} / g\right)^{0.5}
$$

In an actual system, the B blocks would assume a somewhat more expanded conformation, since the surface of the $\mathrm{S}$ core repels the B blocks. However, we neglected this effect and employed eq 11 in our estimation of the $R_{\mathrm{B}}$.

Employing eq 3 and 11 and the values of $N_{m}$ and $d_{\mathrm{S}}$, we estimated the upper and lower concentration $c_{1}{ }^{*}$ and $c_{2}{ }^{*}$. Table II compares $c_{1}{ }^{*}$ and $c_{2}{ }^{*}$ with the rheologically observed $c_{\mathrm{R}}{ }^{*}$ for the SB1 to SB5/C14 micelle systems at $25^{\circ} \mathrm{C} . c_{\mathrm{R}}{ }^{*}$ is roughly equal to or slightly larger than the critical threshold $c_{2}{ }^{*}$. Thermodynamic properties of polymer solutions show a crossover just above the critical threshold. ${ }^{18}$ For example, the concentration dependence of osmotic pressure changes from a virial-expansion type to a scaling-form type as predicted by des Cloizeaux $^{18}$ and experimentally demonstrated by Noda et al. ${ }^{19}$ The driving force of the macrolattice formation may be attributed to local osmotic pressure gradient, and may emerge drastically at the critical threshold.

\section{Relation of Dynamic Behavior with Molecular Dimension}

Dynamic behavior of $10 \mathrm{wt} \% \mathrm{C} 14$ solutions of SB1 to SB5 block copolymer samples were compared. The concentration of $10 \mathrm{wt} \%$ is sufficiently higher than $c_{\mathrm{R}}{ }^{*}$ as shown in Table II, and all systems possess large yield values. Actually, a macrolattice was formed in each system such as shown in Figure 4. At $25^{\circ} \mathrm{C}$, the systems behaved as elastic gels, and steady flow data could not be obtained. Figure 5 shows the dynamic behavior of the systems before yielding. For all systems, the storage modulus $G^{\prime}$ showed plateau regions against frequency typical of viscoelastic solids. The systems were not 


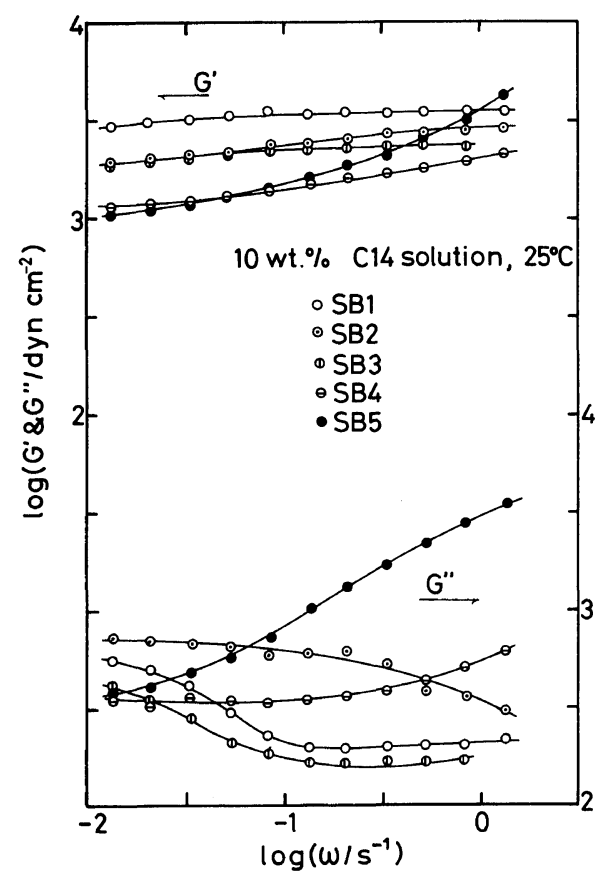

Figure 5. Dynamic behavior of the $10 \mathrm{wt} \% \mathrm{Cl} 14$ solutions of the SB1 to SB5 block copolymers before yielding at $25^{\circ} \mathrm{C}$.

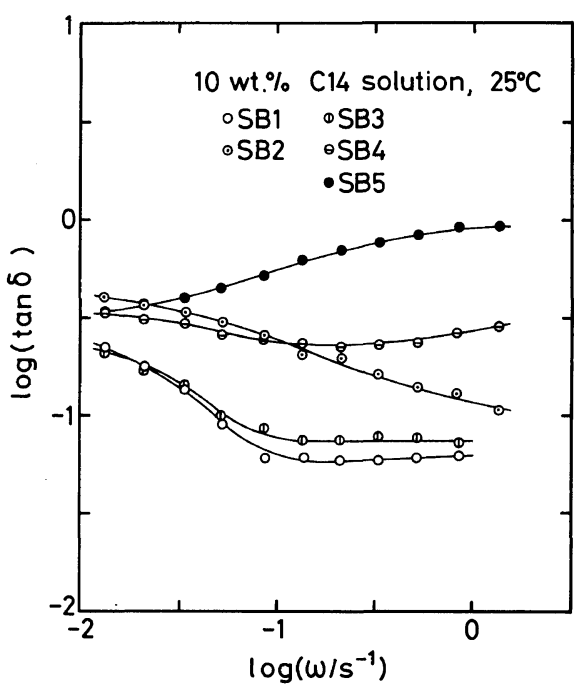

Figure 6. Frequency dependence of the loss tangent $\tan \delta$ of the $10 \mathrm{wt}^{\%} \mathrm{C} 14$ solutions of the SB1 to SB5 block copolymer at $25^{\circ} \mathrm{C}$.

perfectly elastic but possessed small loss moduli $G^{\prime \prime}$. The difference in their rheology becomes more evident when the loss tangent $\tan \delta$ of the systems are compared in Figure 6. The value of $\tan \delta$ of the SB1 system is smaller than that of the SB2 system. The same tendency also appears for the SB3, SB4, and SB5 systems, i.e., $\tan \delta$ increases in the order SB3 < SB4 < SB5. As described in the previous section, SB1 and SB2 have the same $\mathrm{S}$ blocks but the latter has longer $B$ blocks than the former. This situation also holds for the SB3, SB4, and SB5. The difference in $\tan \delta$ is therefore attributed to the difference in the size of the dissolved B blocks. Entanglements of the B blocks may be strongly enhanced as they become longer. An interesting feature of the dynamic behavior of the micelle systems is that the $\tan \delta$ values, especially of SB1 and SB3 which have relatively short B blocks, are very small in comparison with those of homopolymer solutions in the rubbery plateau region. That is, the micelle systems with concentrations just above the $c_{\mathrm{R}}{ }^{*}$ are nearly elastic when subjected to sufficiently small stress. In other words, $G^{\prime}$ is independent of the frequency $\omega$ and $\tan \delta=G^{\prime \prime} / G^{\prime}$ is very small. Such a difference between homopolymer solutions and micelle systems apparently reflects the difference in the stress generating mechanisms of the two systems. In the former stress is generated mostly from intermolecular entanglements, ${ }^{20}$ while in the latter some other mechanism is operative in addition to the deformation of entangled B blocks. This mechanism may be attributed to the macrolattice structure. When strain is imposed on the micelle system, each B block undergoes deformation, and at the same time, the regular arrangement of the micelles (macrolattice) is also deformed. The deformed macrolattice generates elastic stress which does not relax. ${ }^{6}$ These two mechanisms, delayed reptating motions of the entangled B blocks and the deformation of the macrolattice, generate the stress in $\mathrm{SB} / \mathrm{C} 14$ systems above their $c_{\mathrm{R}}{ }^{*}$. The contribution of the second mechanism to the whole stress becomes dominant at low frequency and low concentration close to $c_{\mathrm{R}}{ }^{*}$. We can see this situation for the SB1 and SB2 systems in Figure 5. The B block is shorter in the former and thus generate less loss of mechanical energy due to entanglement interactions. The macrolattice has shorter Bragg spacing as shown in Table III, and is more rigid in the former for storing more elastic energy.

\section{Relation between Critical Temperature and Molecular Dimension}


Figure 7 shows the effect of temperature on the steady flow behavior of the SB1/C14(10) system obtained at 100,120 , and $135^{\circ} \mathrm{C}$. The system clearly exhibits plastic flow at 100 and $120^{\circ} \mathrm{C}$, while Newtonian viscous flow at above $135^{\circ} \mathrm{C}$. A transition from plastic to viscous behavior also appeared in the dynamic measurements at the same temperature, which we call the critical temperature $T^{*}$. As described in the previous paper, ${ }^{6}$ this transition of rheology well corresponds to the disordering of the macrolattice. The correspondence of the rheological and structural transitions provides an understanding of the transition temperature in terms of the macrolattice structure. The concentration fluctuation in the matrix $B$ (plus solvent

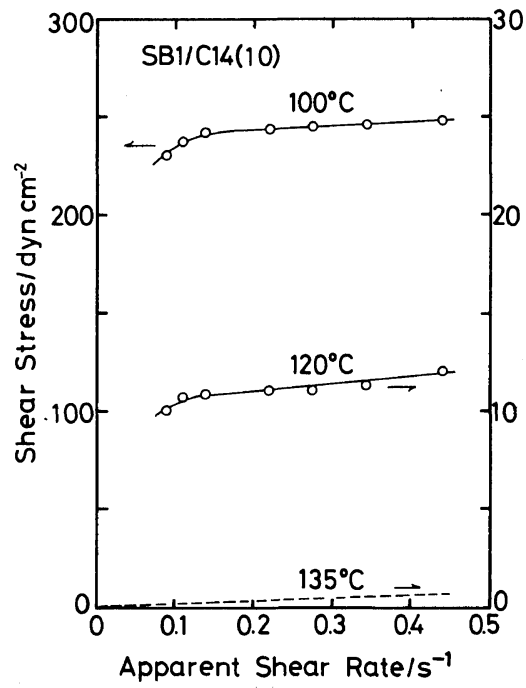

Figure 7. Steady flow behavior of the SB1/C14(10) system obtained at 100,120 , and $135^{\circ} \mathrm{C}$.
C14) phase becomes larger because of the enhanced segmental motions of B blocks at high temperature. Beside this, the mixing of the $\mathrm{S}$ and $\mathrm{B}$ blocks takes place to some extent. The lattice-disordering may possibly result from these changes. However, the problem of how and why the lattice-disordering occurs has not been completely solved.

To shed some light on this problem, we carried out steady flow and dynamic measurements at various temperatures, and the critical temperature $T^{*}$ was determined for other SB2 to SB5 block copolymer systems. Table IV compares $T^{*}$ of $10 \mathrm{wt} \% \mathrm{C} 14$ solutions of SB1 to SB5. When the molecular weight of the $\mathrm{S}$ block $M_{\mathrm{S}}$ is fixed, $T^{*}$ becomes higher with decreasing length of the B block $M_{\mathrm{B}}$ (compare SB1 and SB2, or SB3, SB4, and SB5 systems). Also, when the composition of the blocks is fixed $T^{*}$ becomes higher with increasing block lengths of $M_{\mathrm{S}}$ and $M_{\mathrm{B}}$ (compare SB2 and SB4). These results suggest that diffuse interphase between the $\mathrm{S}$ core and $\mathrm{B}$ matrix appears to play a key role in the lattice-disordering phenomenon. The interphase seems more diffuse at a fixed composition as $M_{\mathrm{S}}$ and $M_{\mathrm{B}}$ decrease. ${ }^{10}$ On the other hand, when $M_{\mathrm{S}}$ is fixed and the $M_{\mathrm{B}}$ increases, the segmental motion of the B blocks seems to be enhanced. This behavior also makes the interphase more diffuse, as long as $M_{\mathrm{B}}$ is considerably larger than $M_{\mathrm{S}}$. The interphase becomes more diffuse on mixing the $\mathrm{S}$ and $\mathrm{B}$ blocks with increasing temperature. Therefore, making smaller the block lengths of $M_{\mathrm{B}}$ and $M_{\mathrm{S}}$ and/or making smaller the $\mathrm{S} / \mathrm{B}$ ratio seems to have the same result as an increase in temperature.

In our previous work, ${ }^{6}$ the cSB sample, which had nearly the same molecular weight and com-

Table IV. Critical temperature $T^{*}$ of (a) the $10 \mathrm{wt} \% \mathrm{C} 14$ solutions of SB1 to SB5 samples and (b) $\mathrm{C} 14$ solutions of $\mathrm{CSB}^{\mathrm{a}}$ sample at various concentrations

\begin{tabular}{cccccc}
\hline & SB1 & SB2 & SB3 & SB4 & SB5 \\
\hline (a) $\begin{array}{l}10 \mathrm{wt} \% \\
\mathrm{C} 14 \text { solutions } \\
T^{*} /{ }^{\circ} \mathrm{C}\end{array}$ & $120-135$ & $100-120$ & $>135$ & $120-135$ & $100-120$ \\
& 10 & 20 & 35 & & \\
(b) $\begin{array}{c}\mathrm{cSB} / \mathrm{C} 14 \\
\mathrm{conc} / \mathrm{wt} \% \\
T^{*} /{ }^{\circ} \mathrm{C}\end{array}$ & $50-60$ & $60-70$ & $80-90$ & & \\
& & $10-70$ & & & \\
\end{tabular}

${ }^{a}$ Solprene 1205 (Phillips Petroleum); $10^{-3} M_{n}=52, \mathrm{~S}$ content $=29.5 \mathrm{wt} \%$, block S content $=18.2 \mathrm{wt} \%{ }^{1}{ }^{1}(c f$. Table I for the SB data). 
position as SB1 but a slightly tapered portion between the $\mathrm{S}$ and $\mathrm{B}$ blocks, exhibited a much lower $T^{*}$ and higher $c_{\mathrm{R}}{ }^{*}$ than those of the $\mathrm{SB} 1 / \mathrm{C} 14$ systems, as can be seen from Table IV. The difference in $T^{*}$ of the two systems may be attributed to the diffuse interphase of cSB system due to its tapered structure. The diffuse-interphase hypothesis will be further examined in our future publications.

Acknowledgements. The authors express their sincere appreciation to Mr. M. Shibayama, Dr. T. Hashimoto, and Professor H. Kawai, Department of Polymer Chemistry, Faculty of Engineering, Kyoto University, for generously carrying out SAXS measurements and making helpful comments.

This study was supported in part by the Ministry of Education, Science and Culture (Mombusho) under grants 347081 and 543026.

\section{REFERENCES}

1. T. Kotaka and J. L. White, Trans. Soc. Rheol., 17, 587 (1973).

2. T. Masuda, Y. Matsumoto, T. Matsumoto, and S. Onogi, Nihon Reoroji Gakkaishi, 5, 135 (1977); T. Masuda, Y. Matsumoto, and S. Onogi, J. Macromol. Sci., Phys., B17, 256 (1980).

3. K. Osaki, B. S. Kim, and M. Kurata, Bull. Inst. Chem. Res., Kyoto Univ., 56, 56 (1978).

4. T. Kotaka, T. Tanaka, and H. Inagaki, Polym. J., 3, 327 (1972); T. Kotaka, T. Tanaka, M. Hattori, and H. Inagaki, Macromolecules, 11, 138 (1978).

5. H. Watanabe and T. Kotaka, Nihon Reoroji Gakkaishi, 8, 26 (1980).
6. H. Watanabe, T. Kotaka, T. Hashimoto, M. Shibayama, and H. Kawai, J. Rheol., 26, 153 (1982).

7. T. Kotaka and H. Watanabe, Nihon Reoroji Gakkaishi, 10, 24 (1982).

8. M. Shibayama, T. Hashimoto, and H. Kawai, Macromolecules, submitted (1982).

9. D. J. Meier, J. Polym. Sci., C, 26, 81 (1969); Prep. Polym. Colloq., Soc. Polym. Jpn., 1, 83 (1977).

10. E. Helfand and Z. R. Wasserman, Macromolecules, 9, 879 (1976); ibid., 11, 960 (1978).

11. H. Watanabe and T. Kotaka, J. Rheol., submitted (1982).

12. M. Shibayama, T. Hashimoto, H. Kawai, H. Watanabe, and T. Kotaka, Macromolecules, submitted (1982).

13. C. Sadron and B. Gallot, Macromol. Chem., 164, 301 (1973).

14. M. Fujimura, T. Hashimoto, and H. Kawai, Mem. Fac. Eng., Kyoto Univ., 43(2), 224 (1981); T. Hashimoto, S. Suehiro, M. Shibayama, K. Saijo, and H. Kawai, Polym. J., 13, 501 (1981).

15. see, for example, P. G. de Gennes, "Scaling Concepts in Polymer Physics," Cornell University Press, London, 1979, Chapter 3.

16. M. Kurata, Y. Tsunashima, M. Iwama, and K. Kamada, "Viscosity-Molecular Weight Relationships and Unperturbed Dimensions of Linear Chain Molecules," in J. Brandrup and E. $H$. Immergut, Ed., "Polymer Handbook," 2nd ed, Wiley-Interscience, New York, 1975.

17. see, for example, P. J. Flory, "Statistical Mechanics of Chain Molecules," John Wiley \& Sons, New York, 1968.

18. J. des Cloizeaux, J. Phys. (Paris), 36, 281 (1975).

19. I. Noda, N. Kato, T. Kitano, and M. Nagasawa, Macromolecules, 14, 668 (1981).

20. see, for example, J. D. Ferry, "Viscoelastic Properties of Polymers," John Wiley \& Sons, New York, 1969. 